\title{
Predictive Analytics of COVID-19 Pandemic: Statistical Modelling Perspective
}

\author{
S Lokesh KUMAR*, Vergin Raja SAROBIN M and Jani ANBARASI L
}

Vellore Institute of Technology, Chennai, India

("Corresponding author’s e-mail: hifilokesh@gmail.com)

Received: 3 December 2020, Revised: 26 March 2021, Accepted: 31 March 2021

\begin{abstract}
The novel Coronavirus-19 (COVID-19) is an infectious disease and it causes serious lung injury. COVID-19 induces human disease, which has killed numerous people around the world. Moreover, the World Health Organization (WHO) declares this virus as a pandemic and all countries attempt to monitor and control it by locking all places. The illness induces respiratory influenza like problems with symptoms such as cold, cough, fever, and the difficulty of breathing in extremely severe cases. COVID-2019 has been viewed as a global pandemic, and a few analyses are being performed using multiple computational methods to predict the possible development of this pestilence. Considering the various conditions and inquiries these numerical models are based on future tendency. Multiple techniques have been proposed that could be helpful in forecasting the spread of COVID-19. Through statistical modeling on the COVID-19 data, we performed linear regression, random forest, ARIMA and LSTMs, to estimate the empirical indication of COVID-19 ailment and intensity in 4 countries (USA, India, Brazil, and Russia), in order to come up with a better validation.
\end{abstract}

Keywords: COVID-19 forecasting, Machine learning, Regression, Time series prediction, Deep learning, Statistical modelling

\section{Introduction}

According to the WHO, COVID-19 is a contagious illness that has been accountable in infecting millions of citizens and killing thousands of people around the world since its $1^{\text {st }}$ outbreak [1,2]. Due to the effect of the COVID-19 pandemic on population and the global economic growth, policymakers and citizens are particularly worried about when the COVID-19 epidemic peaks [3]. Furthermore, Boccaletti et al. [4] found 3 research groups who could participate in combating the current pandemic:

- The group of applied mathematicians, geneticists and physicians, designing advanced models of dissemination to the basic characteristics of a particular pathogen.

- The group of convoluted system scientists researching the propagation of diseases using techniques and concepts from mathematical mechanics and nonlinear dynamics.

- The group of scientists using Artificial Intelligence (AI), and more precisely, machine learning pathways to create detailed forecasting behavior.

The effects of COVID-19 on population, through forecasts of possible events, and also factors of willingness to assist to explain the transmission of this virus are also examined in numerous studies [5]. In addition, observational studies in time series forecasting serves an important role in the global health sector as it helps administrators to establish risk management to deter future epidemics. Because of its influence on the healthcare system, predicting outbreaks as reliably as possible is critical. Application of $\mathrm{AI}$ and computational intelligence techniques are diversified in various areas such as e-healthcare [6,7], smart city [8], data processing [9], predictive maintenance [10], etc. Likewise, application of AI based 
techniques play a vital role in this research work. In order to guarantee precision, AI models have been commonly used over the years to estimate empirical time series. Furthermore, a review of advances in COVID-19 data processing in the AI context was proposed by Vaishya et al. [11].

The precision of conventional prediction relies primarily on the availability of evidence to build its projections. In influenza outbreaks there are little statistics at all and then limited as time goes on, leaving forecasts largely unpredictable. A New York Times article [12] warned about crisis peaking on February 18,2020 , even though nearly 50 days had elapsed before the virus was detected. In addition, there are fears that the statistics could not be accurate, as was the case with Severe Acute Respiratory Syndrome (SARS) where the people infected and deaths was given falsely to mask the severity of the outbreak. Similarly, the documentation also did not represent the right numbers as a new group of "clinically diagnosed" was added to that of "lab-confirmed" on February 13 [13]. Such issues limit the accuracy of predictions and raise ambiguity, making it more difficult to draw definitive conclusions. Linked to predicting precision and volatility, the understanding of epidemics and pandemics has to do with a more serious issue. Politicians are concerned with the steps to be taken when the general public is nervous with their health/lives affecting the outbreak.

In addition, the vaccine industry is working with a strong commercial interest on vaccines for the new virus. During the period of SARS, as governments were convinced of the seriousness of the virus imported large numbers of vaccines which were never implemented when their dissemination stopped without the need for people to be vaccinated. The major challenge is of course the liabilities and the unfounded fear and its potential disastrous effects, as what occurred with the Spanish flu of 1918. In comparison, SARS resulted in the deaths of 774 people in 2003, and bird flu claimed about 100 in 1997. About the same period, there is much less worry over the flu, that affects over 646,000 individuals each year [14]. Health forecasts are also not reliable when significantly underestimating their uncertainty [15]. It is also more difficult to forecast the likelihood of epidemics and pandemics, since there are limitations in calculating the number of cases to be investigated. Amid the inaccuracies associated with medical forecasts, forecasting is still crucial in order to better explain the present situation, and on how to plan ahead. In this article, we include predictive predictions using accurate time series models for reported COVID-19 cases, and we analyze the history of recovered cases.

\section{Literature survey}

The patterns and the statistical time series data are present in most business and economic related statistics. In making critical business decisions it is mandatory to forecast patterns and seasonal trends accurately. There are several methods used to estimate or forecast the pattern data and the seasonal time sequence. The most popular and simple approach involves extracting the seasonal variations through the data using certain methods of trend adjustment. Other typical approaches include models with BoxJerkins and model with Autoregressive Integrated Moving Average (ARIMA) [16]. The ARIMA methodology is implemented after the input series has been transformed to a stationary time series. However, the fundamental mechanism used in the ARIMA model is of linear form, thus, the other nonlinear patters are not captured in the time series.

Nowadays, capital exchange and product sourcing fields are essential to trading and decisionmaking. Here, the key role is the future stock market data analysis and fund creation. Using traditional statistical data, many machine learning approaches were used to estimate information from the financial data set to minimize risk mitigation, enabling the expected return on capital, and create investments accurately. Using data from the financial time series, several techniques such as Artificial Neural Networks (ANN), Evolutionary Algorithms (EA), Support Vector Machines (SVM) and Fuzzy Logic Systems (FLS) have been used for market exploration and investment creating applications. A Fuzzy evaluation model [17] and a Fuzzy rule-based approach were built by Chu et al. and Zargham et al. to construct portfolios based on selected securities, respectively. 
Yan and Chowdhury correlated mid-term electricity-based Market Clearing Price (MCP) forecasting with Vector Support Machine (SVM) and Least Squares Support Vector Machine (LSSVM) [18]. The advantage of using SVM above other prediction approaches is that the challenges can be eliminated in SVM such as fitting data, large types of sample data, and limited local issues. Compared with other complex approaches used in ANN and Bayesian networks, the method of SVM learning is much simpler and easier to model. The authors endorsed the distinction between SVM and LSSVM forecasting methods by calculating hourly electricity MCP for the mid-term. The dataset is taken from the existing PJM integrated electrical market. Data values from January 1, 2009 to December 31, 2009, minus June data, are known to be training data. The residual data was taken from June 1, 2009 until June 30, 2009 as test data. A difference exists between these 2 methods as they are experimentally implemented. SVM uses a quadratic formula during the training process, while LSSVM performs a linear equation. The next distinction is that the SVM extracts only such information with a fixed factor as a support vector, and the LSSVM extracts the test data as a vector.

Yan and Chowdhury. suggested another MCP-based mid-term forecasting model using the Vector Support System (SVM) and ARMAX [20]. For the experimentation the same PJM correlated data on the electricity dataset was used. Related to current models such as SVM, ARMAX and hybrid LSSVMARMAX [21], the proposed SVM-ARMAX hybrid model is computed. The contrast reveals that the hybrid approach SVM-ARMAX is more reliable than the methods mentioned above. The reason for this is that, by collecting a linear module, the SVM model can achieve improved forecasting accuracy.

Frohlich et al. included a technique of this effect with the help of SVM with GA for forecasting the financial data [22]. Huang et al. later suggested a somewhat closer approach involving simultaneous optimization mechanism and showed that classification performance is better in SVM to some financial data sets of the University of California Irvine (UCI). Huang later suggested a related form of hybrid model for stock selection or analysis based on the learning algorithm used in the SVR which uses the wrapper approach, where different data features are extracted in that approach. The SVR is used to produce the predictions based on the rating of the real stock data. The highly valued inventories are eventually taken for portfolio building. Parallel optimization and simulation of the parameters is performed using GA.

Deep learning argues that in classification or regression tasks a hierarchical model is more effective than the surface models [23]. Recurrent Neural Networks (RNNs) contain hidden states spread over time, and this enables them to collect a great deal of past knowledge, and because of their ability to handle sequential data of varying length [24], they are more widely used in forecasting applications. Whereas, RNN have a significant drawback that they cannot solve the gradient loss and can only collect short-term memory as they only require the previous time step's hidden layer activation functions [25].

\section{Proposed work}

The proposed methods as given in Figure 1 implemented in the paper take into consideration the statistical forecasting models, as well as the state-of-the-art architectures to give a comparative analysis on the forecasting model accuracy. The algorithms that are implemented in this paper are conventional techniques such as linear regression, random forest, ARIMA and deep learning techniques such as Long Short-term Memory networks (LSTMs). Error metrics such as Mean Absolute Error (MAE), Mean Squared Error (MSE), Root Mean Squared Error (RMSE) and R-Squared are performed on each algorithm to find out its accuracy in forecasting cases and deaths, and to compare it with other models. 
http://wjst.wu.ac.th

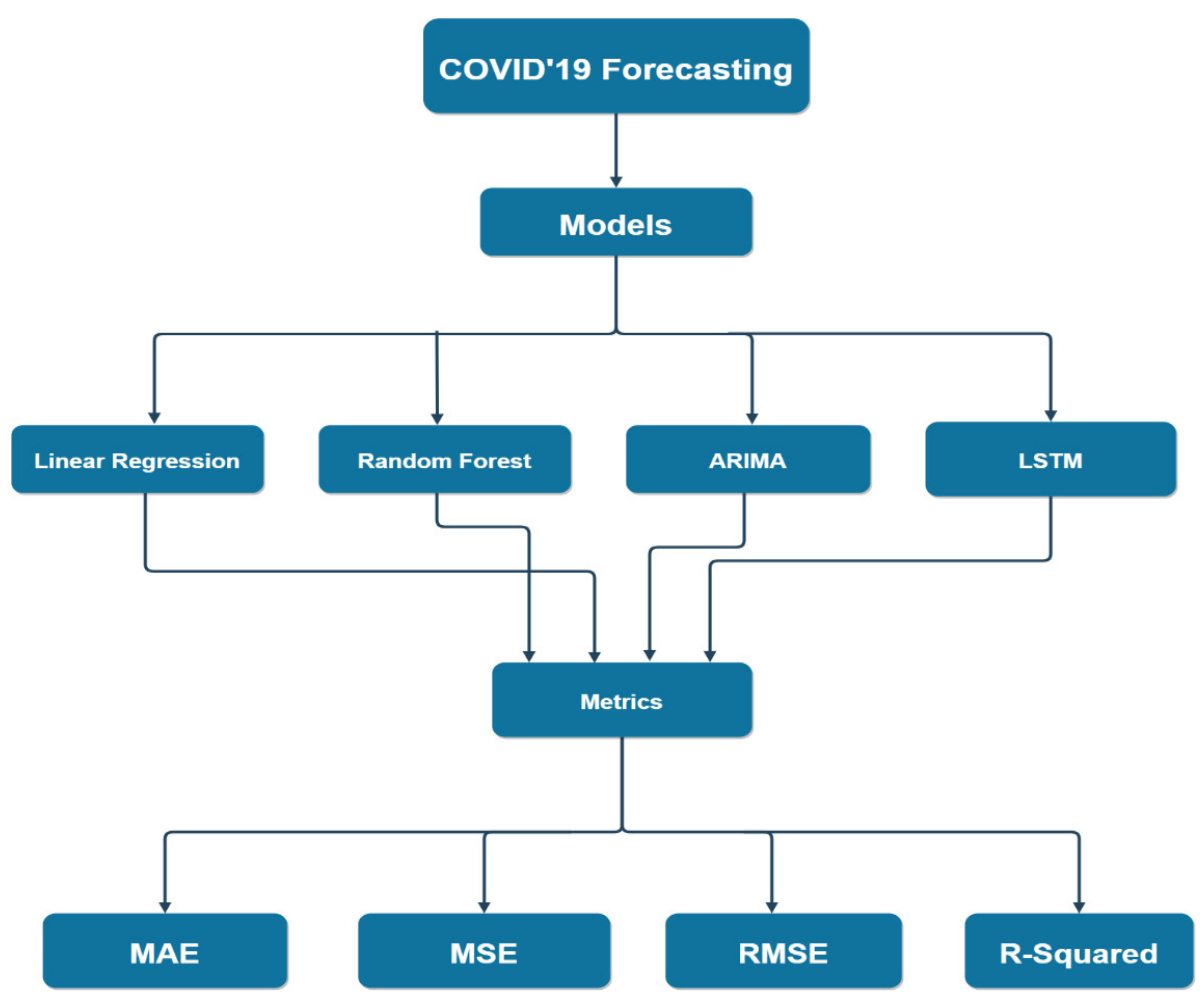

Figure 1 Proposed Framework for COVID-19 Forecasting.

\section{Data source}

The dataset implemented focuses on the cumulative cases of COVID-19 that were confirmed in 4 countries until $25^{\text {th }}$ November of 2020. The dataset was collected from our World in Data by University of Oxford that collects the weekly information of COVID-19 cases from all countries and makes it publicly available. Among all the countries, the 4 taken into consideration for analysis were USA, India, Brazil, and Russia. The state's calculation duration changes as each state counts from the day of the $1^{\text {st }}$ event to the day of the last record. The accumulated reported cases and deaths in each state are shown in the heat map as seen in Figure 1, as well as the time from the $1^{\text {st }}$ and last records. The improvement in the way Health departments pay for the number of incidents will affect the evidence provided in this report.

\section{Machine learning methods}

\section{Linear Regression (LR)}

Linear Regression (LR) is a straightforward way to show the relationship between a dependent variable and at least one independent variable. LR was the principal method of analysis to be extensively developed and commonly used in methodologies (Yan \& Su, 2009). LR demonstrates the relation between 2 variables by applying a straight state to the knowledge centered on them. One factor is perceived as independent, and the other is perceived as dependent. The configuration of the LR1 line is conditional:

$$
Y=b X+a
$$


http://wjst.wu.ac.th

\section{Random Forest (RF)}

Random Forest (RF) is an ensemble of learning algorithms based on methods. RF consists of a series of classifiers for tree. Every tree is composed of nodes and edges. The received group classifies new data points through a majority within each classification model's predictions, as shown in Figure 4. This approach incorporates a bagging cycle (bootstrap aggregation) and a set of random splits. Each tree is extracted from the data set from a separate bootstrap sample, and each tree categorizes the data. The final outcome is a majority vote between the trees.

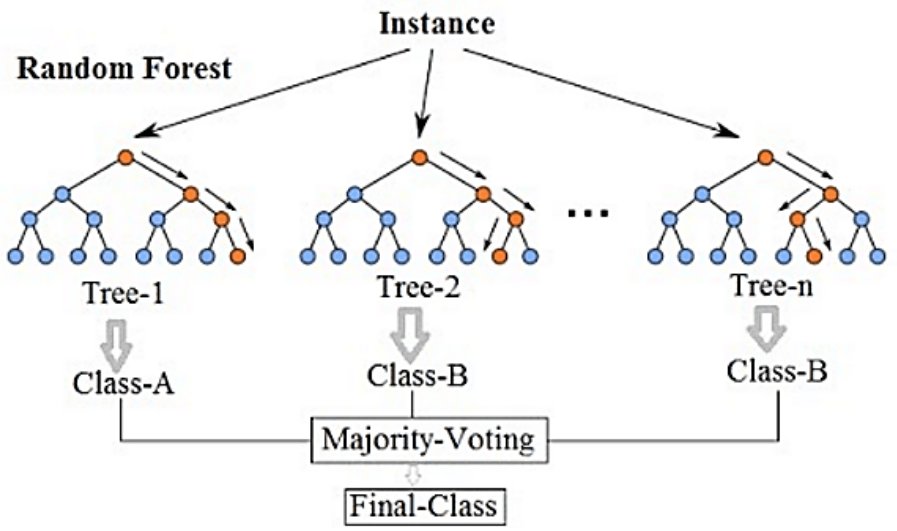

Figure 2 Random forest methodology.

The random forest algorithm is defined by the following steps:

- Construct samples of the data from $\mathrm{k}$ trees bootstrap.

- For each of the bootstrap samples grow an unpruned tree.

- Randomly sample n-try of the predictors at each node, and pick the best split among those factors.

- Predict new data through a combination of the $\mathrm{k}$ tree predictions

\section{ARIMA}

ARIMA is used in stationary time series data to map linear trends. ARIMA is denoted as ARIMA model ( $\mathrm{p}, \mathrm{d}$, and $\mathrm{q})$. The variables $\mathrm{p}$ and $\mathrm{q}$ are respectively in the order of the model AR and the model MA, and $d$ is the degree of differentiation [9]. The ARIMA model can be denoted mathematically as:

$$
y_{t}=\theta_{0}+\varphi_{1} y_{t-1}+\varphi_{2} y_{t-2}+\cdots+\varphi_{p} y_{t-p}+\varepsilon_{t}+\theta_{1} \varepsilon_{t-1}-\theta_{2} \varepsilon_{t-2}-\cdots-\theta_{q} \varepsilon_{t-q}
$$

where $y_{t}$ gives actual value under time $\mathrm{t}, \varepsilon_{t}$ gives the random error at $\mathrm{t}$. The $\varphi_{j}$ and $\theta_{i}$ are the ARIMA model coefficients.

The core principle produced by ARIMA is that with constant variance, the error series observes zero mean, which implements the i.i.d condition. Constructing for a given dataset can be defined in 3 steps:

- Series recognition (stationarity)

- Parameter estimation and partial autocorrelation plots are used to pick component values

- Model diagnostic testing (finding the 'right' fitting prediction model using AIC \& BIC) 
http://wjst.wu.ac.th

\section{Deep learning methods-LSTM}

LSTMs are regarded one of the most viable options for forecasting activities, and they simulate possible predictions based on the different features outlined in the dataset. The data moves with LSTMs via elements known as cell states. LSTMs may properly recall items, or forget them. Information obtained over progressive time frames is represented as time series data and LSTMs are commonly suggested to be a reliable approach to generate forecasts of these data values. The paradigm moves the past veiled state into the corresponding stage of the structure in this sort of architecture as shown in Figure 3.

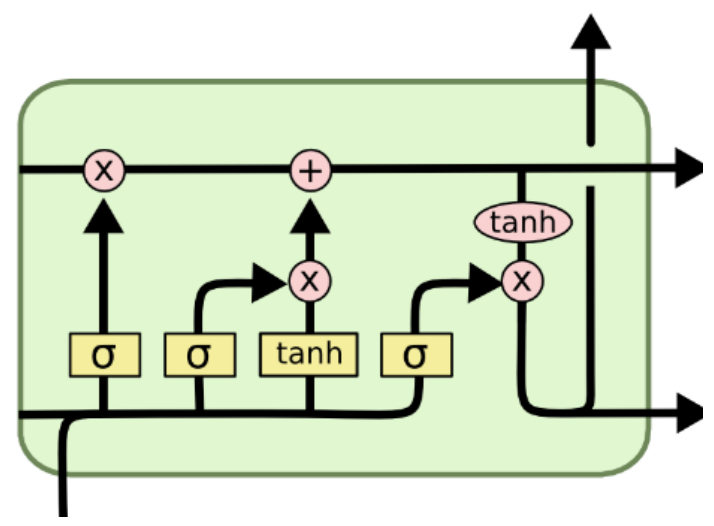

Figure 3 LSTM memory cell: The repeating module in an LSTM contains 4 interacting layers.

Long-term storage of short-term memory cells (LSTM) [21] together with RNNs is used because RNNs can only store a limited amount of information. The issues of the gradient disappearing [22] which afflicts RNN are solved by LSTMs. LSTM cells are similar to RNN cells with replaceable memory blocks for hidden modules. Figure 3 represents the LSTM memory block with the entry, forget, and exit gates. This mechanism prevents the brain cell from producing information accumulated over many phases of time [23]. It is possible to mathematically express the state of the gates as given by:

$i_{t}=\sigma\left(W_{x i} x_{t}+W_{h i} h_{t-1}+W_{c i} c_{t-1}+b_{i}\right)$

$f_{t}=\sigma\left(W_{x f} x_{t}+W_{h f} h_{t-1}+W_{c f} c_{t-1}+b_{f}\right)$

$c_{t}=i_{t} \tanh \left(W_{x c} x_{t}+W_{h c} h_{t-1}+b_{c}\right)+f_{t} c_{t-1}$

$o_{t}=\sigma\left(W_{x o} x_{t}+W_{h o} h_{t-1}+W_{c o} c_{t}+b_{o}\right)$

$h_{t}=o_{t} \tanh \left(c_{t}\right)$

In Eqs. (3) to (7) i, o, f, c is the input gate, exit gate, forget gate, cell, and $\sigma$ is the same scale logistic sigmoid activation function as the hidden vector. The weight matrices where $W_{c i}$ describes the vector of the cell input gate are represented by $\mathrm{W}$. Based on the foregoing calculation especially in the present time level, the input gate decides how many data can be transmitted through and defends the cell against insignificant entries. Forget gate determines data which is not applicable from the preceding time period and should be removed. The output gate manages information flow to the whole system. With these, either design improvements are made in LSTM or secret layers, are primarily intended for performance enhancement. 
http://wjst.wu.ac.th

\section{Results and discussion}

This section summarizes the effects of the scripts produced in forecasts using testing data. From the collected data, 2 time series were created, forecasting of cases and deaths. In this experiment, it is concluded that each of these 3 sequences has a duration, that is to say, the outbreak finally ends. The amount of reported cases is clearly proportional to the amount of suspected cases recorded, excluding the recovered cases and deaths.

- In a time-series model, the residuals are what is left over when a device is fitted. For certain (but not all) time series models, the residuals are proportional to the variance between the measurements and the fitted values in question:

$e_{t}=y_{t}-y_{t}^{\wedge}$

Residuals are helpful in testing whether a model has collected the details in the data correctly. A good method of forecasting gives residuals with the following properties:

- If associations occur between residuals, so information is observed in residuals that can be used in forecasting purposes.

- Residuals give zero mean.

- If the residuals are of a value rather than zero, so the projections are skewed.
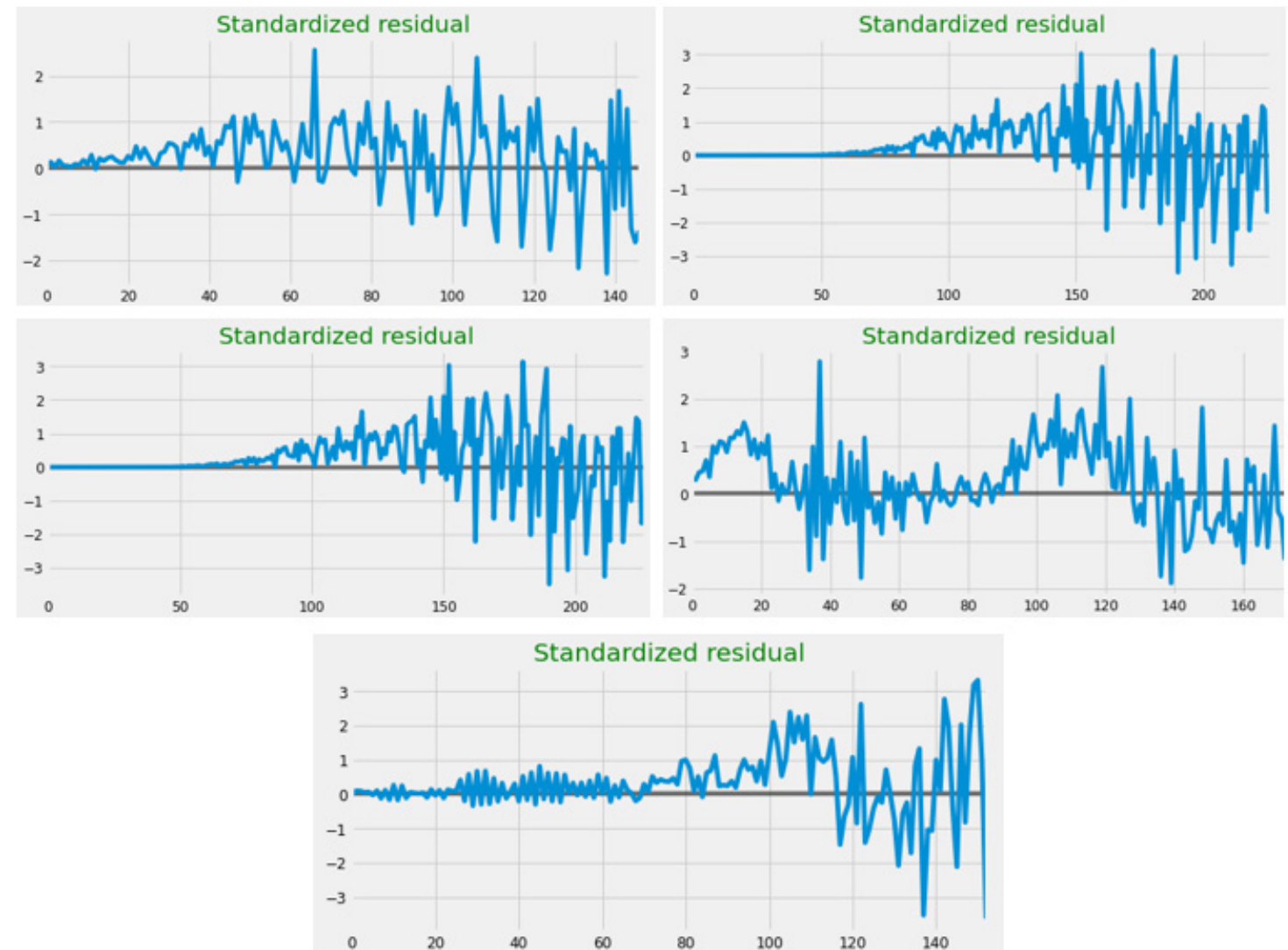

Figure 3 Standardized residual diagnosis for each country: Brazil, Russia, USA, and India (Clockwise). 
http://wjst.wu.ac.th

\section{Total cases forecasting}

This paper further looks into how each of the models perform on the data of particular countries and review the accuracy.

USA: The performance of linear regression and random forest provide comparatively lower results than ARIMA and deep learning LSTM model. The RMSE scores are given in Table 1. ARIMA provides a good forecasting result with AIC score 3696.007, but lesser than LSTM as shown in Figure 3.

India: The overall performance of all algorithms has comparatively higher accuracy rate than other dataset. The linear regression and random forest algorithms give RMSE of 218514.082 and 156308.794 respectively. ARIMA scores 2998.166 on AIC and 3019.865 on BIC. The prediction analysis for LSTM and ARIMA can be seen in Figure 3 below.

Brazil: The RMSE scores are taken into consideration for evaluation. From the statistical models implemented, linear regression gives better performance compared to random forest model as shown in Table 1 below. Akaike Information Criterion (AIC) and Bayesian information criterion (BIC) are used to evaluate the performance of ARIMA model. The forecasting results of ARIMA and LSTM can be seen in Figure 3.

Russia: The regression RMSE score gives comparatively larger values from the datasets covered above this data and follows different trend with more volatility. ARIMA performs better than the discussed algorithms giving AIC and BIC score of 2679.898 and 2701.682 respectively. LSTMs again outperform the statistical models with a very good fit as shown in Figure 3.

\section{USA}

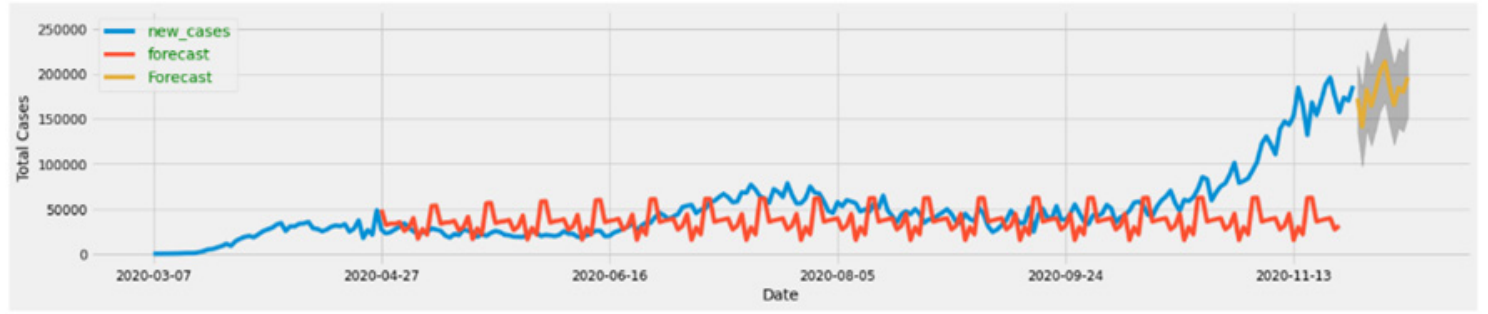

A

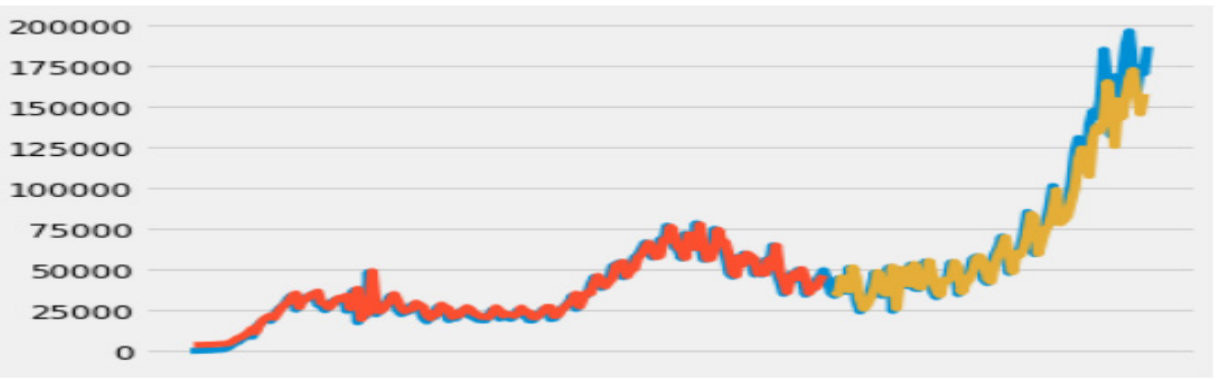

B 


\section{INDIA}

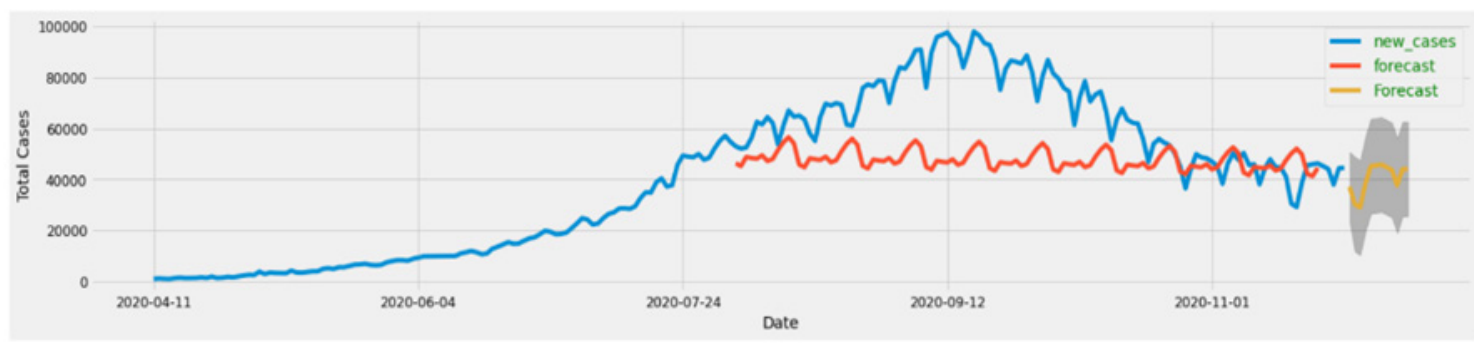

A

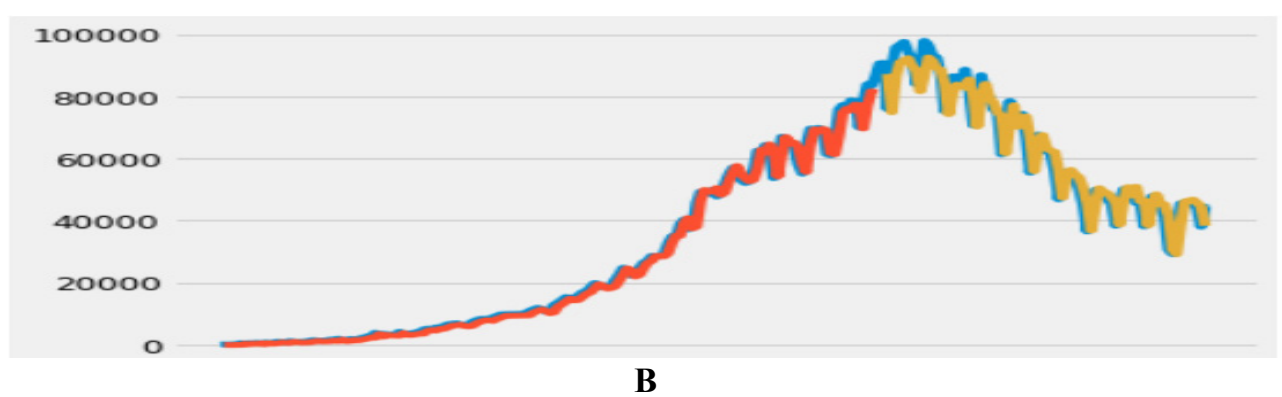

\section{BRAZIL}

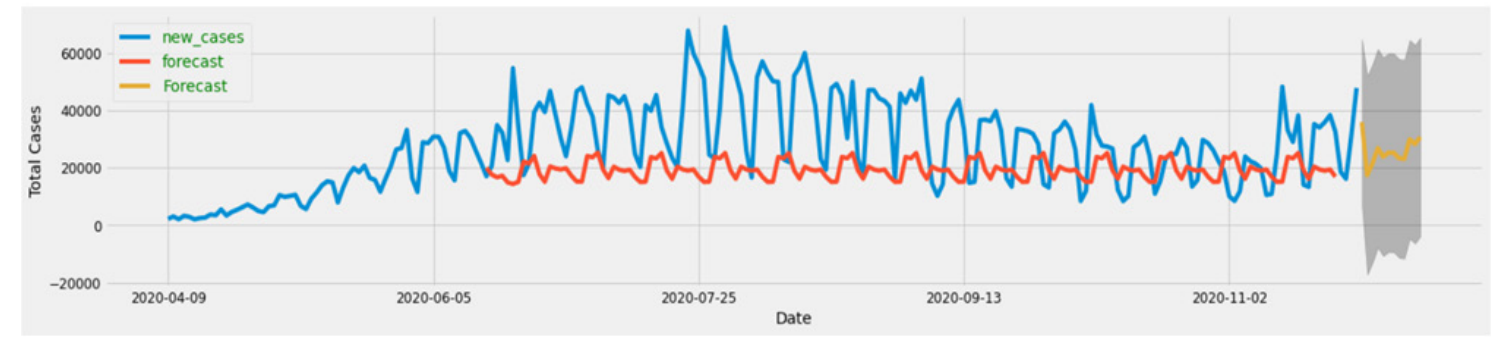

A

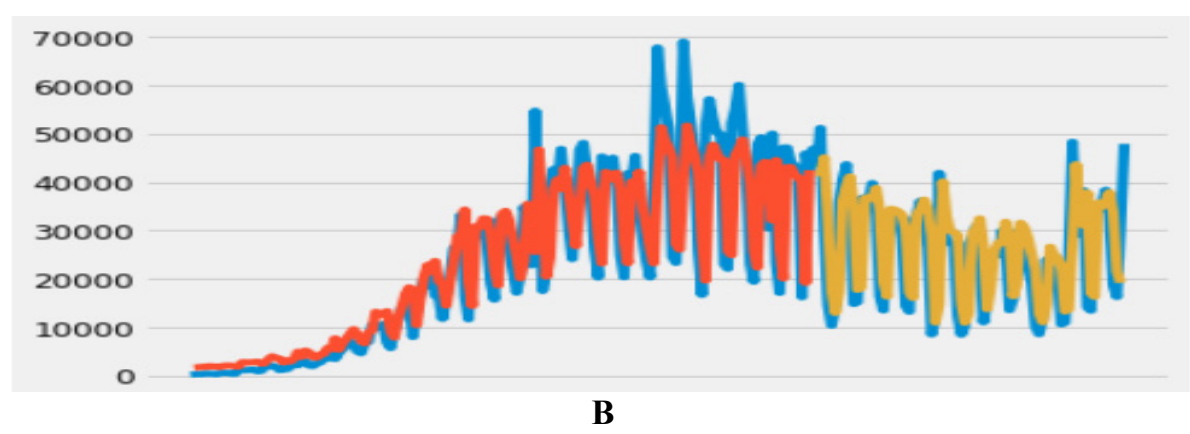




\section{RUSSIA}

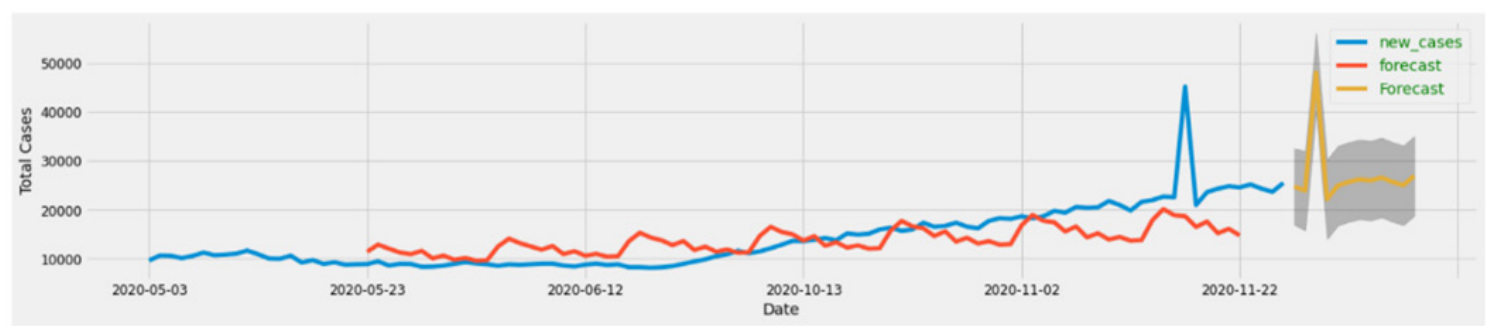

A

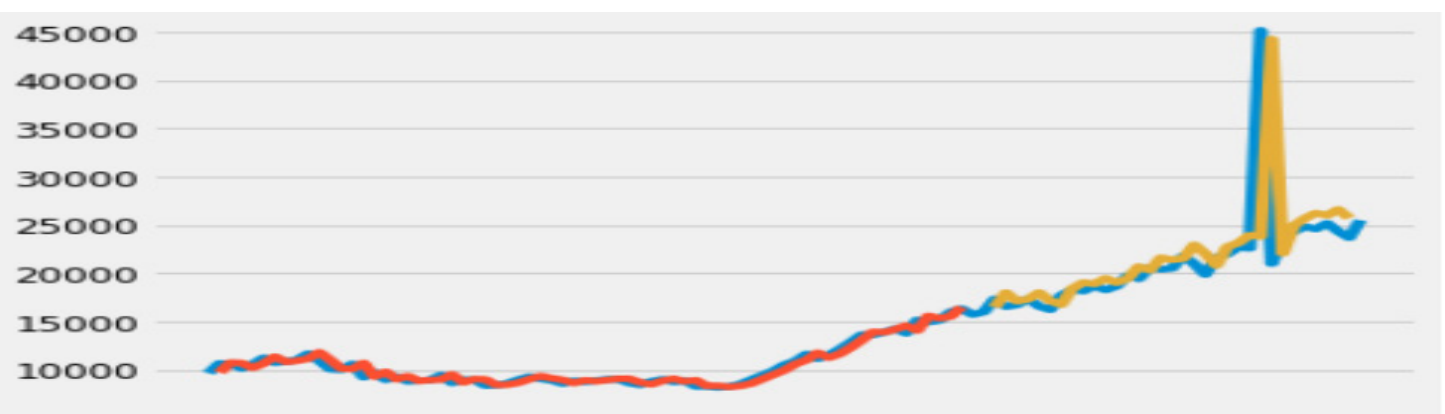

B

Figure 3 Case forecasting results (USA, India, Brazil and Russia): A. ARIMA, B. LSTM.

Table 1 Performance metrics for cases forecasting on Random Forest (RF), Linear Regression (LR) and LSTM.

\begin{tabular}{|c|c|c|c|c|c|c|c|c|c|c|c|c|}
\hline & \multicolumn{3}{|c|}{ Brazil } & \multicolumn{3}{|c|}{ Russia } & \multicolumn{3}{|c|}{ USA } & \multicolumn{3}{|c|}{ India } \\
\hline & RF & LR & LSTM & $\mathbf{R F}$ & LR & LSTM & RF & LR & LSTM & $\mathbf{R F}$ & LR & LSTM \\
\hline MAE & 3525 & 4200 & 2231 & 314 & 1349 & 309 & 3122 & 10063 & 3220 & 1133 & 1621 & 484 \\
\hline MSE & 52313330 & 67098058 & 38613796 & 1152145 & 3841902 & 110221 & 21204252 & 137687328 & 17842176 & 6429642 & 9752142 & 398161 \\
\hline RMSE & 7232 & 8191 & 6214 & 1073 & 1960 & 332 & 4604 & 11734 & 4224 & 2535 & 3122 & 631 \\
\hline R-squared & 0.82 & 0.77 & 0.92 & 0.92 & 0.72 & 0.92 & 0.66 & 0.60 & 0.82 & 0.98 & 0.91 & 0.99 \\
\hline
\end{tabular}

\section{Total deaths forecasting}

From the above results, it can be clearly concluded that ARIMA based forecasting showed significantly better forecasting results as compared to other machine learning models (linear regression and random forest). It is further recommended to look into ARIMA and LSTMs closely to validate variability across death forecasting for each country for comparative analysis purposes. 
$\underline{\text { USA }}$

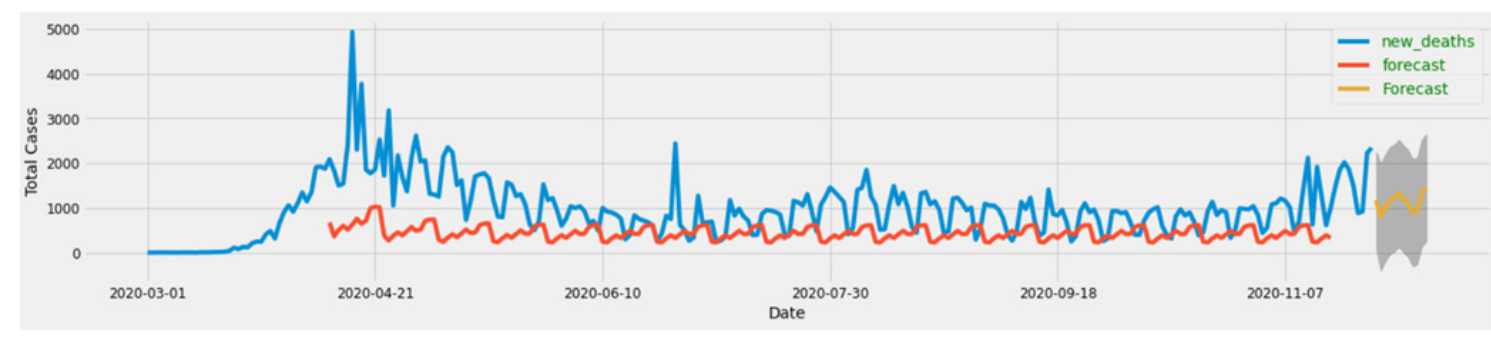

$\mathbf{A}$

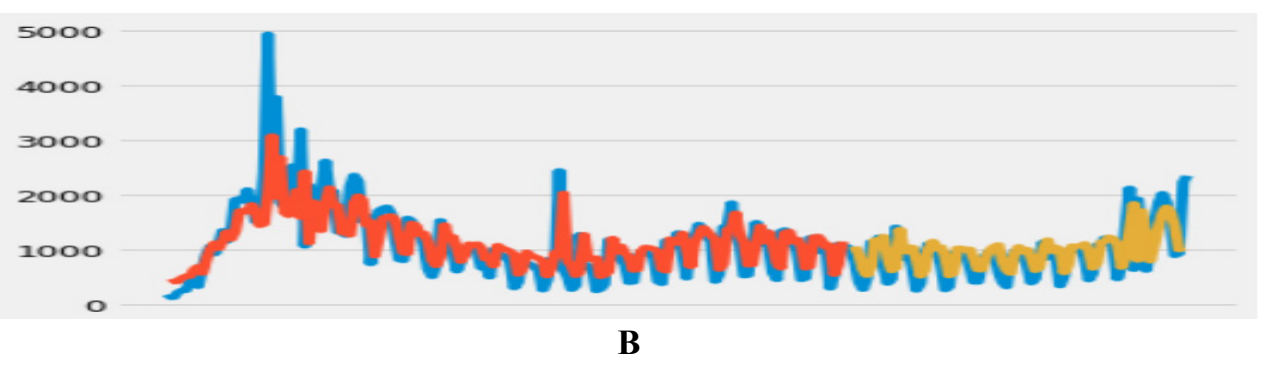

INDIA

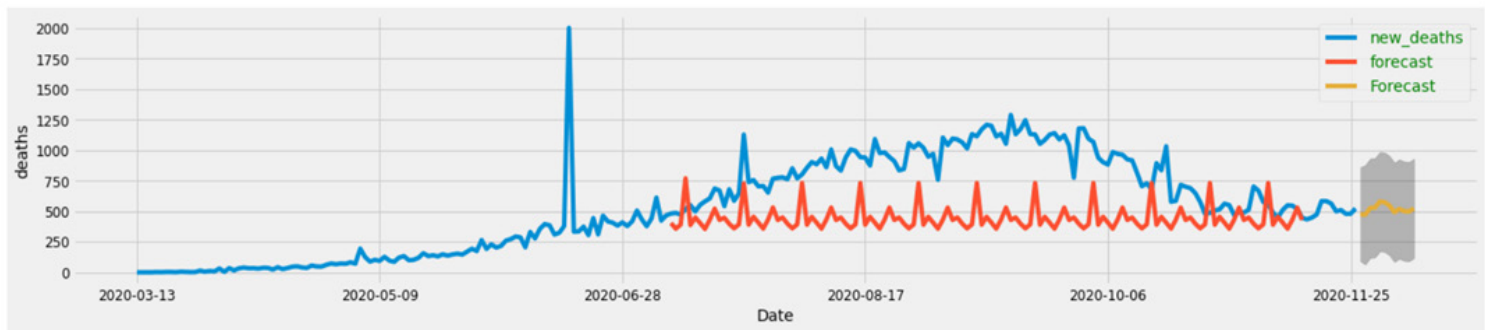

A

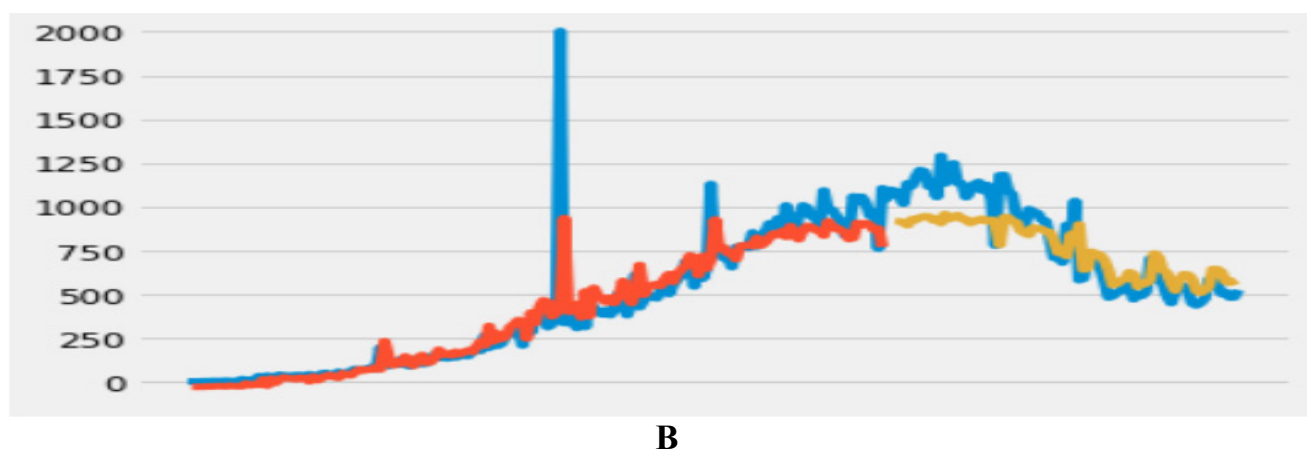




\section{BRAZIL}

A
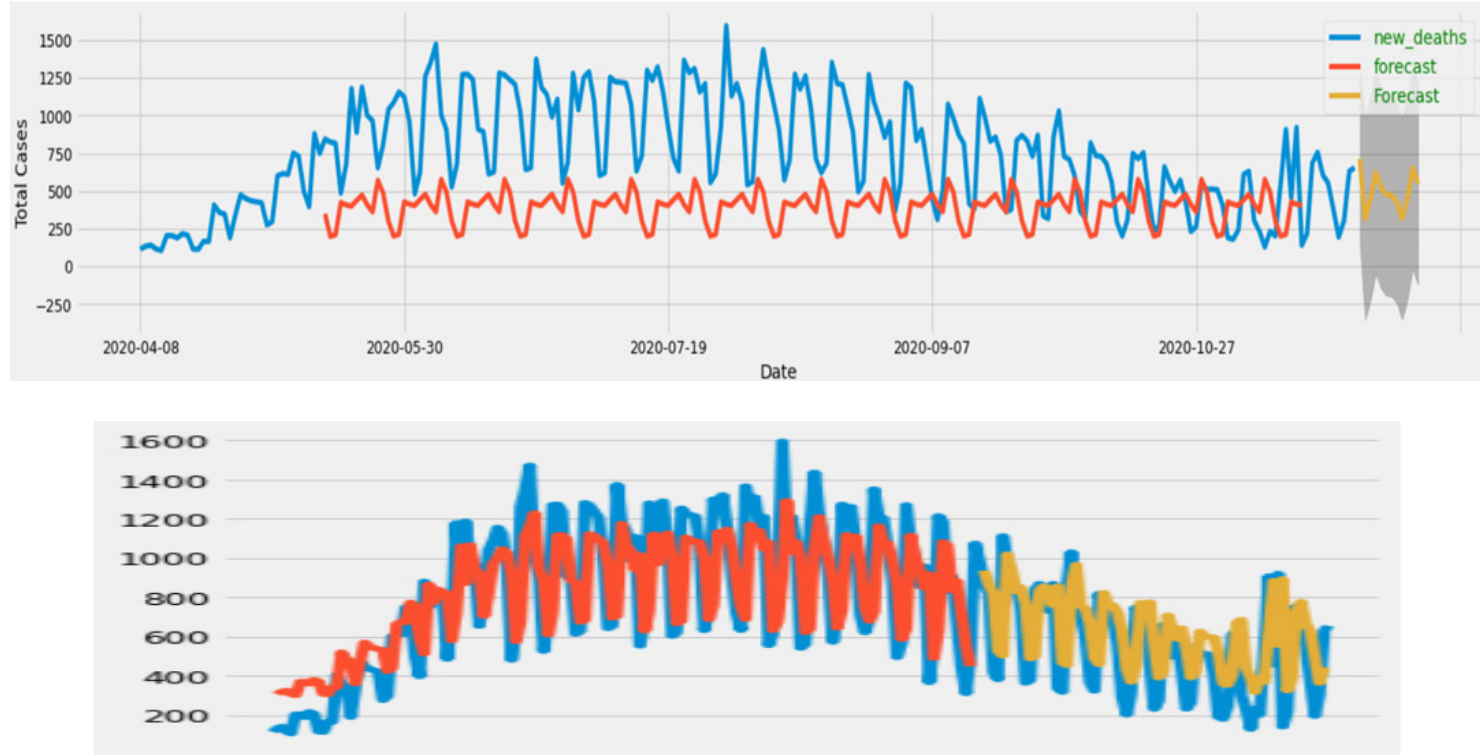

B

RUSSIA

A
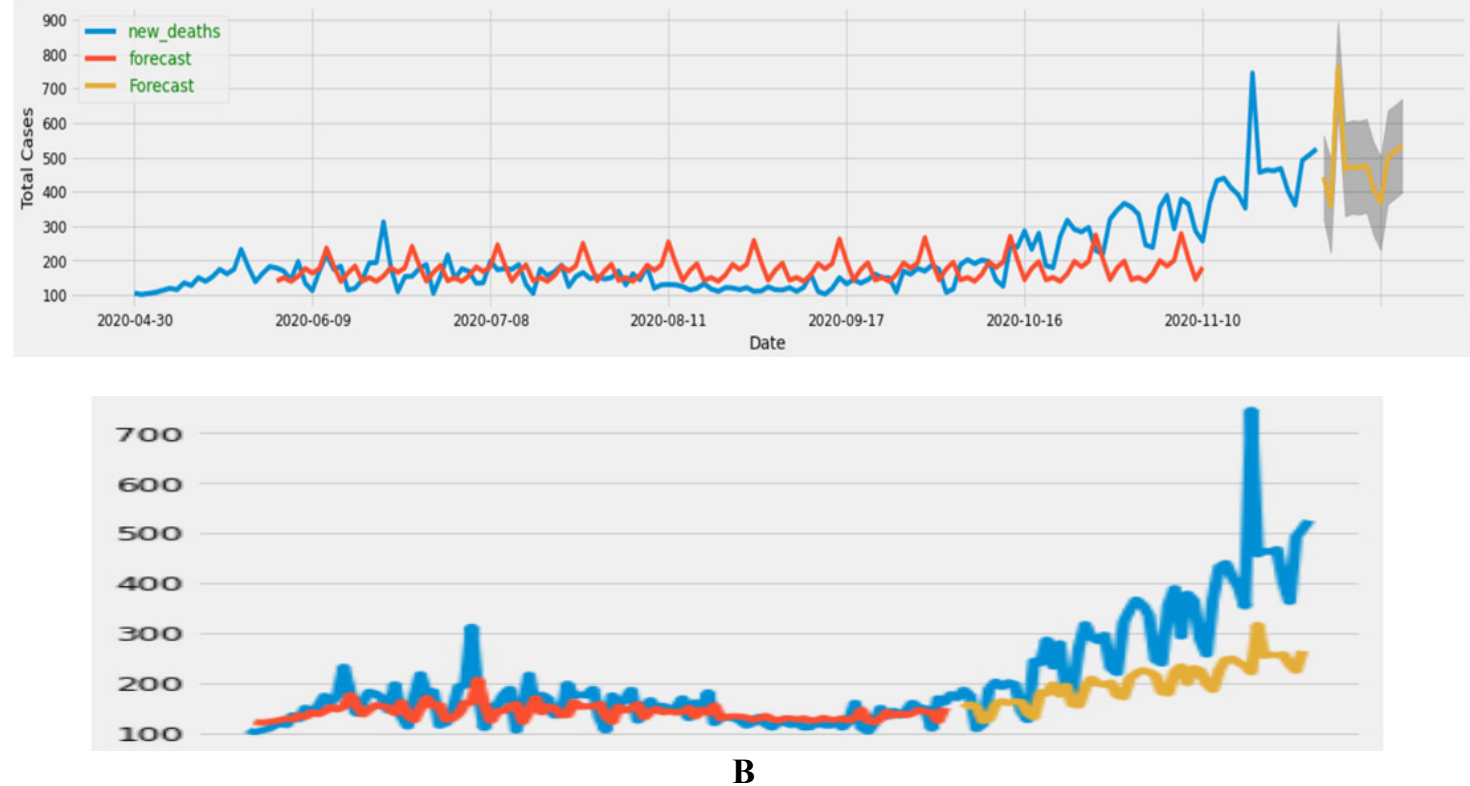

Figure 4 Death forecasting results (USA, India, Brazil, and Russia): A. ARIMA, B. LSTM. 
http://wjst.wu.ac.th

From Figure 4, it is clearly distinguishable from the forecasting results that LSTM outperforms ARIMA statistical model in every country dataset. The LSTM results also showcase better fitting in training as well as testing data compared to ARIMA model.

Table 2 Performance metrics for death forecasting on Random Forest (RF), Linear Regression (LR) and LSTM.

\begin{tabular}{|c|c|c|c|c|c|c|c|c|c|c|c|c|}
\hline & \multicolumn{3}{|c|}{ Brazil } & \multicolumn{3}{|c|}{ Russia } & \multicolumn{3}{|c|}{ USA } & \multicolumn{3}{|c|}{ India } \\
\hline & RF & LR & LSTM & RF & LR & LSTM & RF & LR & LSTM & RF & LR & LSTM \\
\hline MAE & 77.07 & 159.66 & 52 & 15.65 & 1449.61 & 21 & 226.75 & 510.32 & 220 & 24.47 & 61.13 & 25 \\
\hline MSE & 17586.8 & 39487.3 & 10404 & 950.8 & 1449.55 & 900 & 188456.9 & 493048.2 & 180625 & 1916.9 & 5429.2 & 1849 \\
\hline RMSE & 132.61 & 198.714 & 102 & 30.83 & 38.073 & 30 & 434.11 & 702.173 & 425 & 43.782 & 73.683 & 43 \\
\hline R-squared & 0.929 & 0.842 & 0.938 & 0.818 & 0.723 & 0.949 & 0.725 & 0.282 & 0.778 & 0.983 & 0.952 & 0.988 \\
\hline
\end{tabular}

\section{Conclusions}

The COVID-19 has caused respiratory ailments in humans and created long term damage in many cases too. Apart from this, there has been several cases of deaths around the world. In this paper, 3 statistical modelling approaches (linear regression, random forest, and ARIMA) and 1 deep learning approach (LSTMs) were explored. It is aimed on providing a comparative analysis taking into consideration the dataset from Our World in Data of 4 countries (Brazil, India, Russia, and USA), to provide optimum test cases and validation across multiple trend patterns focused at 2 forecasting events namely, cases forecasting and deaths forecasting. Many performance metrics and diagnostic tools such as residuals, correlograms, RMSE, AIC and BIC were implemented to monitor models' accuracy. With respect to the results obtained, out of the statistical approaches taken, ARIMA outperformed linear regression and random forest in terms of accuracy prediction of test data. ARIMA and LSTMs were compared again with death forecasting task, in which LSTMs were able to provide very high accuracy in comparison. In the future works, it is meant to adopt novel deep learning algorithms such as CNNs and RNNs along with hyperparameter optimization which will be able to help in accurate monitoring of the future cases of COVID-19.

\section{References}

[1] C Sohrabi, Z Alsafi, N O’Neill, M Khan, A Kerwan, A Al-Jabir, C Iosifidis and R Agha. World health organization declares global emergency: A review of the 2019 novel coronavirus (COVID19). Int. J. Surg. 2020; 76, 71-6.

[2] X Zhang, R Ma and L Wang. Predicting turning point, duration and attack rate of COVID-19 outbreaks in major Western countries. Chaos Solitons Fractals 2020; 135, 109829.

[3] S Boccaletti, W Ditto, G Mindlin and A Atangana. Modeling and forecasting of epidemic spreading: The case of COVID-19 and beyond. Chaos Solitons Fractals 2020; 135, 109794.

[4] D Fanelli and F Piazza. Analysis and forecast of COVID-19 spreading in China, Italy and France. Chaos Solitons Fractals 2020; 134, 109761.

[5] JK Davis, T Gebrehiwot, M Worku, W Awoke, A Mihretie, D Nekorchuk and MC Wimberly. A genetic algorithm for identifying spatially-varying environmental drivers in a malaria time series model. Environ. Model. Softw. 2019; 119, 275-84.

[6] MVR Sarobin, S Alphonse, M Gupta and T Joshi. Rapid eye movement monitoring system using artificial intelligence techniques. In: Proceedings of the International Conference on Information Management \& Machine Intelligence, Singapore. 2019, p. 605-10.

[7] A Gondalia, D Dixit, S Parashar, V Raghava and A Sengupta. IoT-based healthcare monitoring system for war soldiers using machine learning. Procedia Comput. Sci. 2018; 133, 1005-13. 
http://wjst.wu.ac.th

[8] MVR Sarobin and R Ganesan. Swarm intelligence in wireless sensor networks: A survey. Int. J. Pure Appl. Math. 2015; 101, 773-807.

[9] S Vasudevan, N Chauhan, V Sarobin and S Geetha. Image-based recommendation engine using VGG model. In: Proceedings of the Advances in Communication and Computational Technology, Singapore. 2021, p. 257-65.

[10] A Chazhoor, Y Mounika, MVR Sarobin, MV Sanjana and R Yasashvini. Predictive maintenance using machine learning based classification models. IOP Conf. Ser. Mater. Sci. Eng. 2020; 954, 012001.

[11] R Vaishya, M Javaid, IH Khan and A Haleem. Artificial intelligence (AI) applications for COVID19 pandemic. Diabetes Metab. Syndr. 2020; 14, 337-9.

[12] Wang V. Coronavirus epidemic keeps growing, but spread in China slows. New York Times, Available at: https://www.nytimes.com/2020/02/18/world/asia/china-coronavirus-cases.html? referringSource=articleShare, accessed February 2020.

[13] BBC. Coronavirus: Sharp increase in deaths and cases in Hubei, Available at: https://www.bbc.co.uk/news/worldasia-china-51482994, accessed February 2020.

[14] Medicine Net. Flu kills 646,000 people worldwide each year: Study finds, Available at: https://www.medicinenet.com/script/main/art.asp?articlekey=208914, accessed February 2020.

[15] S Makridakis, A Wakefield and R Kirkham. Predicting medical risks and appreciating uncertainty. Foresight 2019; 52, 28-35.

[16] KH Jacobsen. Will COVID-19 generate global preparedness? Lancet 2020; 395, 1013-4.

[17] TC Chu, CT Tsao and YR Shiue. Application of fuzzy multiple attribute decision making on company analysis for stock selection. In: Proceedings of the Soft Computing in Intelligent Systems and Information Processing. Proceedings of the 1996 Asian Fuzzy Systems Symposium, Kenting, Taiwan. 1996, p. 509-14.

[18] X Yan and NA Chowdhury. Midterm electricity market clearing price forecasting using two-stage multiple support vector machine. J. Energy 2015; 2015, 384528.

[19] S Zhang, M Diao, W Yu, L Pei, Z Lin and D Chen. Estimation of the reproductive number of novel coronavirus (COVID-19) and the probable outbreak size on the diamond princess cruise ship: A data-driven analysis. Int. J. Infect. Dis. 2020; 93, 201-4.

[20] X Yan and NA Chowdhury. Mid-term electricity market clearing price forecasting utilizing hybrid support vector machine and auto-regressive moving average with external input. Int. J. Electr. Power Energy Syst. 2014; 63, 64-70.

[21] IAWA Razak, IZ Abidin, YK Siah, AAZ Abidin, TKA Rahman, N Baharin and MH Jali. An optimization method of genetic algorithm for LSSVM in medium term electricity price forecasting. $J$. Telecommun. Electron. Comput. Eng. 2018; 10,99-103.

[22] H Frohlich, O Chapelle and B Scholkopf. Feature selection for support vector machines by means of genetic algorithm. In: Proceedings of the $15^{\text {th }}$ IEEE International Conference on Tools with Artificial Intelligence, Sacramento, CA, USA. 2003, p. 142-8.

[23] HI Fawaz, G Forestier, J Weber, L Idoumghar and PA Muller. Deep learning for time series classification: A review. Data Min. Knowl. Discov. 2019; 33, 917-63.

[24] M Längkvist, L Karlsson and A Loutfi. A review of unsupervised feature learning and deep learning for time-series modeling. Pattern Recognit. Lett. 2014; 42, 11-24.

[25] JCB Gamboa. Deep learning for time-series analysis. ArXiv 2017. Available at: https://arxiv.org/abs/1701.01887, accessed February 2020. 